\title{
SUBORDINATION-PRESERVING INTEGRAL OPERATORS
}

\author{
BY
}

\author{
SANFORD S. MILLER, ${ }^{1}$ PETRU T. MOCANU AND MAXWELL O. READE ${ }^{1}$
}

ABSTRACT. Let $\beta$ and $\gamma$ be complex numbers and let $H$ be the space of functions regular in the unit disc. Subordination of functions $f, g \in H$ is denoted by $f \prec g$. Let $K \subset H$ and let the operator $A: K \rightarrow H$ be defined by $F=A(f)$, where

$$
F(z)=\left[\frac{1}{z^{\gamma}} \int_{0}^{z} f^{\beta}(t) t^{\gamma-1} d t\right]^{1 / \beta} .
$$

The authors determine conditions under which

$$
f \prec g \Rightarrow A(f) \prec A(g),
$$

and then use this result to obtain new distortion theorems for some classes of regular functions.

I. Introduction. Let $f(z)$ and $g(z)$ be regular in the unit disc $U$. We say that $f(z)$ is subordinate to $g(z)$, written $f(z) \prec g(z)$ or $f \prec g$, if there exists a function $w(z)$ regular in $U$ which satisfies $w(0)=0,|w(z)|<1$ and $f(z)=g(w(z))$. If $g(z)$ is univalent in $U$, then $f \prec g$ if and only if $f(0)=g(0)$ and $f(U) \subset g(U)[9$, p. 35].

Let $H=H(U)$ be the space of regular functions defined in $U$ and let $K \subset H$. Letting $A$ be an operator $A: K \rightarrow H$, in this paper we consider conditions under which

$$
f \prec g \Rightarrow A(f) \prec A(g) .
$$

That is, under what conditions is the operator $A$ subordination-preserving? In this paper we shall describe several classes of integral operators for which (1) is satisfied.

Some examples of subordination-preserving integral operators have already appeared in the literature. In $1953 \mathrm{G}$. M. Goluzin [1] considered the operator $A$ : $\{f \in H \mid f(0)=0\} \rightarrow H$ defined by $F=A(f)$, with

$$
F(z)=\int_{0}^{z} \frac{f(t)}{t} d t
$$

He showed that if $g(z)$ is convex $\left(\operatorname{Re}\left[1+z g^{\prime \prime}(z) / g^{\prime}(z)\right]>0\right)$ then $(1)$ is satisfied. In 1970 T. Suffridge [12, p. 777] extended this result to the case when $g(z)$ is starlike $\left(\operatorname{Re}\left[z g^{\prime}(z) / g(z)\right]>0\right)$. In 1975 D. Hallenbeck and S. Ruscheweyh [2, p. 192] showed that (1) holds if $g$ is convex, $\gamma \neq 0, \operatorname{Re} \gamma \geqslant 0$ and $A: H \rightarrow H$ is defined by

Received by the editors May 8, 1983. Presented at the 90th annual meeting of the American Mathematical Society in Louisville, Kentucky, January 27, 1984.

1980 Mathematics Subject Classification. Primary 30C80; Secondary 30C25, 45P05, 30C45.

Key words and phrases. Subordination, integral operator, Loewner chain, univalent function.

${ }^{1}$ This work was carried out while these authors were U.S.A.-Romania Exchange Scholars. 
$F=A(f)$, with

$$
F(z)=z^{-\gamma} \int_{0}^{z} f(t) t^{\gamma \cdots 1} d t
$$

Another proof of this last result is given in [5, Example (b)].

In this paper we consider the integral operator $A: K \rightarrow H$ defined by $A(f)=F$, with

$$
A(f)(z)=F(z)=\left[z^{-\gamma} \int_{0}^{z} f^{\beta}(t) t^{\gamma-1} d t\right]^{1 / \beta} .
$$

We show that this operator is subordination-preserving on appropriate subsets $K \subset H$, for suitable complex constants $\beta$ and $\gamma$. As one application of these results we obtain several new distortion theorems for some classes of regular functions.

II. Preliminaries. We will make use of the following four lemmas. The first two concern differential subordinations, more general forms of which, plus some applications, may be found in [4 and 5].

LEMma 1 [4, p. 291]. Let $G(z)$ be regular and univalent on $\bar{U}$, and let $F(z)$ be regular in $U$ with $F(0)=G(0)$. Suppose there exists a point $z_{0} \in U$ such that $F\left(|z|<\left|z_{0}\right|\right) \subset G(U)$ and $F\left(z_{0}\right)=G\left(\zeta_{0}\right)$, with $\left|\zeta_{0}\right|=1$. Then $z_{0} F^{\prime}\left(z_{0}\right)=m \zeta_{0} G^{\prime}\left(\zeta_{0}\right)$, where $m \geqslant 1$.

LEMmA 2 [4, p. 298]. Let $p(z)=\beta+p_{1} z+\cdots$ be regular in $U$ with $p(z) \not \equiv \beta$ and $\operatorname{Re} \beta>0$. Let the function $\Psi: C^{2} \rightarrow C$ satisfy

(a) $\Psi$ is continuous in a domain $D$ of $C^{2}$,

(b) $(\beta, 0) \in D$ and $\operatorname{Re} \Psi(\beta, 0)>0$,

(c) $\operatorname{Re} \Psi($ ir, s) $\leqslant 0$ when $(i r, s) \in D$, and

$$
s \leqslant-|\beta-i r|^{2} /(2 \operatorname{Re} \beta) \text { for real } r, s .
$$

If $\left(p(z), z p^{\prime}(z)\right) \in D$ when $z \in U$, and $\operatorname{Re} \Psi\left(p(z), z p^{\prime}(z)\right)>0$ when $z \in U$, then $\operatorname{Re} p(z)>0$ for all $z \in U$.

The next lemma concerns subordination (or Loewner) chains. A function $L(z, t)$, $z \in U, t \geqslant 0$, is a subordination chain if $L(\cdot, t)$ is regular and univalent in $U$ for all $t \geqslant 0, L(z, \cdot)$ is continuously differentiable on $R^{+}$for all $z \in U$, and $L(z, s) \prec$ $L(z, t)$ when $0 \leqslant s \leqslant t[9$, p. 157].

LeMma $3[9, \mathrm{p} .159]$. The function $L(z, t)=a_{1}(t) z+\cdots$, with $a_{1}(t) \neq 0$ for all $t \geqslant 0$, is a subordination chain if and only if

$$
\operatorname{Re}\left[z \frac{\partial L}{\partial z} / \frac{\partial L}{\partial t}\right]>0
$$

for $z \in U$ and $t \geqslant 0$.

The last lemma is a modification of a result of K. Sakaguchi [10, Corollary 3]; it provides a sufficient condition for univalence. 
LEMMA 4. Let $\operatorname{Re} \beta>-\frac{1}{2}$, and for $h(z)$ regular in $U$, with $h^{\prime}(0) \neq 0$, let

$$
J(\beta, h) \equiv(\beta-1) \frac{z h^{\prime}(z)}{h(z)}+\left(1+\frac{z h^{\prime \prime}(z)}{h^{\prime}(z)}\right) .
$$

If $\operatorname{Re} J(1, h)>-\frac{1}{2}$, or $\operatorname{Re} J(\beta, h)>-\frac{1}{2}$ when $\beta \neq 1$ and $h(0)=0$, then $h(z)$ is univalent in $U$.

Before we establish our main results, we define the subsets $K$ of $H$ on which the integral operator $A$ given by (2) is defined. Let $\beta$ and $\gamma$ be complex constants with $\operatorname{Re} \beta>0$ and $\operatorname{Re} \gamma \geqslant 0$. Let $K_{\beta, \gamma}$ be defined as follows:

$$
K=K_{\beta, \gamma}=\left\{\begin{array}{l}
H \quad \text { if } \beta=1, \gamma \neq 0, \\
\{f \in H \mid f(0)=0\} \quad \text { if } \beta=1, \gamma=0, \\
\left\{f \in H \mid f(z)=z^{j} h(z), h(z) \neq 0, j \geqslant 1\right\} \quad \text { if } 1 / \beta \in N-\{1\}, \\
\left\{f \in H \mid f(0)=0, f^{\prime}(0) \neq 0, \operatorname{Re}\left[\beta z f^{\prime} / f+\gamma\right]>0\right\} \quad \text { otherwise. }
\end{array}\right.
$$

A straightforward examination of the first three cases shows that $A$ as given by (2) is well defined. That $A$ is well defined in the last case is shown in [6, Corollary 1.1].

III. Main Results. Our first result deals with the special subclass $K_{\beta, 0}$, where $\beta>0$. Note that $K_{1,0}$ is the set of regular functions with $f(0)=0 ; K_{1 / n, 0}$ with $n \in N-\{1\}$ is the set of regular functions $f(z)=z^{j} h(z)$, where $h(z) \neq 0$ and $j \geqslant 1$; and $K_{\beta, 0}$ with $1 / \beta \notin N$ is the class of starlike functions.

THEOREM 1. Let $f \in K_{\beta, 0}$, with $\beta>0$, and let $g(z)=b_{1} z+b_{2} z^{2}+\cdots$ be starlike in $U$. If the operator $A: K_{\beta, 0} \rightarrow H$ is defined by $F=A(F)$, where

$$
F(z)=A(f)(z)=\left[\int_{0}^{z} f^{\beta}(t) t^{-1} d t\right]^{1 / \beta}
$$

then $f \prec g \Rightarrow A(f) \prec A(g)$.

Proof. Equation (4) can be rewritten as the following equation:

$$
F(z)\left[\beta \frac{z F^{\prime}(z)}{F(z)}\right]^{1 / \beta}=f(z) .
$$

The function $G(z) \equiv A(g)(z)$ is an $\alpha$-convex function with $\alpha=1 / \beta$, and hence is regular and univalent in $U$ [7, Theorem 5]. We now show $F(z) \prec G(z)$.

Case $1 . G(z)$ is regular and univalent on $\bar{U}$.

If $F \nless 5 G$ then there exists a $z_{0} \in U$ such that $F\left(|z|<\left|z_{0}\right|\right) \subset G(U)$ and $F\left(z_{0}\right)=$ $G\left(\zeta_{0}\right)$, with $\left|\zeta_{0}\right|=1$. By applying Lemma 1 we find $z_{0} F^{\prime}\left(z_{0}\right)=m \zeta_{0} G^{\prime}\left(\zeta_{0}\right)$, where $m \geqslant 1$. Using this in (5) together with the fact that $g(U)$ is starlike we obtain

$$
f\left(z_{0}\right)=G\left(\zeta_{0}\right)\left[\frac{\beta m \zeta_{0} G^{\prime}\left(\zeta_{0}\right)}{G\left(\zeta_{0}\right)}\right]^{1 / \beta}=m^{1 / \beta} g\left(\zeta_{0}\right) \notin g(U) .
$$

But this contradicts $f \prec g$ and so we must have $F \prec G$. 
Case 2. $G(z)$ is regular and univalent in $U$.

If we let $f_{r}(z)=f(r z)$ and $g_{r}(z)=g(r z)$, for $0<r<1$, then $F(r z)=A\left(f_{r}\right)(z)$ and $G(r z)=A\left(g_{r}\right)(z)$. Since $f_{r} \prec g_{r}$ and $G(r z)$ is regular and univalent in $\bar{U}$ we can apply the results of Case 1 to obtain $F(r z) \prec G(r z)$ for $0<r<1$. By letting $r \rightarrow 1^{-}$ we obtain $F(z) \prec G(z)$. This completes our proof of the theorem.

If we let $\beta=1$ in Theorem 1 we obtain the result of Suffridge [12, p. 777] already noted above. We now consider other special cases and obtain the following distortion theorems.

COROllary 1.1. Let $f(z)$ be regular in $U$ with $f(z)=z^{j} h(z), h(z) \neq 0$ and $j \geqslant 1$. If $f(z) \prec z /(1+z)^{2}$ then:

(a)

$$
\left[\int_{0}^{z} \frac{\sqrt{f(t)}}{t} d t\right]^{2} \prec[2 \arctan \sqrt{z}]^{2}
$$

and

(b)

$$
-\frac{\pi}{2}<-2 \arctan \sqrt{\rho} \leqslant \operatorname{Re} \int_{0}^{z} \frac{\sqrt{f(t)}}{t} d t \leqslant 2 \arctan \sqrt{\rho} \leqslant \frac{\pi}{2},
$$

where $|z|=\rho<1$.

Proof. If we use $\beta=\frac{1}{2}$ and $g(z)=z /(1+z)^{2}$ in Theorem 1 then $G(z)=$ $A(g)(z)=(2 \arctan \sqrt{z})^{2}$ and result (a) is an immediate consequence of the theorem. If $(p(z))^{2} \prec(q(z))^{2}$ then $p(U) \subset[-q(U)] \cup[q(U)]$. Using this last result together with result (a) and the convexity of $g(\rho z)=2 \arctan \sqrt{\rho z}, 0<\rho<1$, we obtain the result (b).

We note that $g(U)=\mathbf{C}-\left\{\left[\frac{1}{4}, \infty\right)\right\}$, the image of $U$ via the Koebe function, while $w=G(z)$ is an $\alpha$-convex function with $\alpha=2$ (and hence convex [7, p. 217]) and $G(U)$ is the interior of the parabola $u=\pi^{2} / 4-v^{2} / \pi^{2}(w=u+i v)$.

COROLlaRY 1.2. If $f(z)$ is starlike and $f(z) \prec z /(1+z)^{2}$ then:

(a) $\left[\int_{0}^{z} f^{2}(t) / t d t\right]^{1 / 2} \prec z /\left[2\left(1+z^{2}\right)\right]^{1 / 2}$,

(b) $\int_{0}^{z} f^{2}(t) / t d t \prec z^{2} / 2\left(1+z^{2}\right)$, and

(c)

$$
\frac{-\rho^{2}}{2\left(1-\rho^{2}\right)} \leqslant \operatorname{Re} \int_{0}^{z} \frac{f^{2}(t)}{t} d t \leqslant \frac{\rho^{2}}{2\left(1+\rho^{2}\right)}<\frac{1}{4},
$$

where $|z|=\rho<1$.

Proof. If we let $\beta=2$ and $g(z)=z /\left(1+z^{2}\right)$ in Theorem 1 , then $G(z)=$ $A(g)(z)=z /\left[2\left(1+z^{2}\right)\right]^{1 / 2}$ and result (a) follows. From (a) and the definition of subordination we obtain

$$
\int_{0}^{z} \frac{f^{2}(t)}{t} d t=\frac{w^{2}(z)}{2\left(1+w^{2}(z)\right)}
$$


where $w(z)$ is regular in $U, w(0)=0$ and $|w(z)|<1$. This last identity yields (b), which then leads to (c).

We note that $g(U)$ is the domain $\mathbf{C}-\left\{\left[\frac{1}{2}, \infty\right) \cup\left(-\infty,-\frac{1}{2}\right]\right\}$ and $G(U)$ is that domain containing the origin which is bounded by the hyperbola $u^{2}-v^{2}=\frac{1}{4}$.

COROLLARY 1.3. If $f(z)=a_{1} z+a_{2} z^{2}+\cdots$ is starlike and if $\operatorname{Re} f^{2}(z)<1$ then:

(a) $\left[\int_{0}^{z} f^{2}(t) / t d t\right]^{1 / 2} \prec\left[\log \left(1+z^{2}\right)\right]^{1 / 2},(\log 1=0)$,

(b) $\int_{0}^{z} f^{2}(t) / t d t \prec \log \left(1+z^{2}\right)$,

(c) $\ln \left(1-\rho^{2}\right) \leqslant \operatorname{Re} \int_{0}^{z} f^{2}(t) / t d t \leqslant \ln \left(1+\rho^{2}\right)<\ln 2$, and

(d)

$$
\frac{-\pi}{2}<-\arctan \frac{\rho^{2}}{\sqrt{1-\rho^{4}}} \leqslant \operatorname{Im} \int_{0}^{z} \frac{f^{2}(t)}{t} d t \leqslant \arctan \frac{\rho^{2}}{\sqrt{1-\rho^{4}}}<\frac{\pi}{2},
$$

where $|z|=\rho<1$.

Proof. The conditions $f(z) \prec q(z)=\sqrt{2} z /\left(1+z^{2}\right)^{1 / 2}$ and $\operatorname{Re} f^{2}(z)<1$ are equivalent. If we use $\beta=2$ and $q(z)=\sqrt{2} z /\left(1+z^{2}\right)^{1 / 2}$ in Theorem 1 then $G(z)=$ $A(g)(z)=\left[\log \left(1+z^{2}\right)\right]^{1 / 2}$ and results (a) and (b) are consequences of the theorem. The function $h(z)=\log \left(1+z^{2}\right)$ is a convex function and we can easily determine the extremal values of $\operatorname{Re} h(r z)$ and $\operatorname{Im} h(r z)$ when $|z|=1$. Combining these results with (b) we obtain the inequalities (c) and (d).

One example of the power of this last corollary occurs if we take $f(z)=$ $(4 \arctan z) / \pi$. Here $f(z) \prec \sqrt{2} z /\left(1+z^{2}\right)^{1 / 2}$, and hence

$$
\begin{gathered}
\int_{0}^{z} \frac{(\arctan t)^{2}}{t} d t \prec \frac{\pi^{2}}{16} \log \left(1+z^{2}\right), \\
\frac{\pi^{2}}{16} \ln \left(1-\rho^{2}\right) \leqslant \operatorname{Re} \int_{0}^{z} \frac{(\arctan t)^{2}}{t} d t \leqslant \frac{\pi^{2}}{16} \ln \left(1+\rho^{2}\right) \leqslant \frac{\pi^{2}}{16} \ln 2,
\end{gathered}
$$

and

$$
\begin{aligned}
\frac{-\pi^{3}}{32} & <\frac{-\pi^{2}}{16} \arctan \frac{\rho}{\sqrt{1-\rho^{4}}} \leqslant \operatorname{Im} \int_{0}^{z} \frac{(\arctan t)^{2}}{t} d t \\
& \leqslant \frac{\pi^{2}}{16} \arctan \frac{\rho}{\sqrt{1-\rho^{4}}} \leqslant \frac{\pi^{3}}{32} .
\end{aligned}
$$

In our second main theorem we assume $\beta$ and $\gamma$ are complex constants, with $\operatorname{Re} \beta>0$ and $\operatorname{Re} \gamma \geqslant 0$, and we make use of the following constants:

$$
\delta_{0}=\frac{1}{2} \frac{|\beta+\gamma|-|\beta-\bar{\gamma}|}{|\beta+\gamma|+|\beta-\bar{\gamma}|}=\frac{2 \operatorname{Re} \beta \operatorname{Re} \gamma}{(|\beta+\gamma|+|\beta-\gamma|)^{2}}
$$

and

$$
\delta=\operatorname{Min}\left(\operatorname{Re} \gamma, \delta_{0}\right) .
$$

Note that $0 \leqslant \delta_{0} \leqslant \frac{1}{2}, 0 \leqslant \delta \leqslant \frac{1}{2}$, and $\delta=\delta_{0}=0$ when $\operatorname{Re} \gamma=0$. 
THEOREM 2. Let $\operatorname{Re} \beta>0, \operatorname{Re} \gamma \geqslant 0$ and $f, g \in K_{\beta, \gamma}$ with $g^{\prime}(0) \neq 0$ and let

$$
\operatorname{Re}\left[(\beta-1) \frac{z g^{\prime}(z)}{g(z)}+\left(1+\frac{z g^{\prime \prime}(z)}{g^{\prime}(z)}\right)\right]>-\delta .
$$

If the operator $A: K_{\beta, \gamma} \rightarrow H$ is defined by $F=A(f)$, where

$$
F(z)=A(f)(z)=\left[\frac{1}{z^{\gamma}} \int_{0}^{z} f^{\beta}(t) t^{\gamma-1} d t\right]^{1 / \beta},
$$

then $f \prec g \Rightarrow A(f) \prec A(g)$.

Proof. Since $-\delta \geqslant-1 / 2$, it follows from (8) and Lemma 4 that the function $g$ is univalent.

If we let $G=A(g)$, then from (9) we obtain

$$
G(z)\left[\beta z \frac{G^{\prime}(z)}{G(z)}+\gamma\right]=g(z) .
$$

We now show that $G$ is univalent in $U$. According to Lemma 4 we can obtain the univalency of $G$ by showing that

$$
\operatorname{Re} J(\beta, G)>0,
$$

and $G^{\prime}(0) \neq 0$ when $\beta=1$, or $G^{\prime}(0) \neq 0$ and $G(0)=0$ when $\beta \neq 1$. These normalization conditions on $G$ follow from $G=A(g)$ and the fact that $g$ satisfies each of them. If we let $P(z)=\beta^{-1} J(\beta, G(z))$, then $P(0)=1$ and we can prove (11) by showing that $\operatorname{Re} \beta P(z)>0$. By logarithmically differentiating (10) twice we obtain

$$
P(z)+\frac{z P^{\prime}(z)}{\beta P(z)+\gamma}=\beta^{-1} J(\beta, g(z)),
$$

where $J(\beta, g)$ is defined by (3). Since $g(z)$ is univalent in $U$, the function $J(\beta, g(z))$ will be regular in $U$. From (7) and (8) we obtain $\operatorname{Re}\left[\beta\left(\beta^{-1} J(\beta, g(z))\right)+\gamma\right]>0$. Hence, as we have shown elsewhere [6, Theorem 1], the solution $P(z)$ of the Briot-Bouquet differential equation (12) is regular and satisfies $\operatorname{Re}[\beta P(z)+\gamma]>0$. Multiplying both sides of (12) by $\beta$ and letting $p(z)=\beta P(z)(=J(\beta, G(z)))$ we obtain

$$
p(z)+\frac{z p^{\prime}(z)}{p(z)+\gamma}=J(\beta, g(z)),
$$

where $p(z)$ is regular in $U, p(0)=\beta$ and $\operatorname{Re}[p(z)+\gamma]>0$. Combining this with (8) we obtain

$$
\operatorname{Re}\left[p(z)+\frac{z p^{\prime}(z)}{p(z)+\gamma}+\delta\right]=\operatorname{Re} \psi\left(p(z), z p^{\prime}(z)\right)>0,
$$

where $\psi(r, s)=r+s /(r+\gamma)+\delta$.

We now use Lemma 2 to prove that $\operatorname{Re} p(z)>0 . \operatorname{Re} \psi(\beta, 0)=\operatorname{Re}(\beta+\delta)>0$, and so we only need to show that $\operatorname{Re} \psi(i r, s) \leqslant 0$, when $s \leqslant-|\beta-i r|^{2} /(2 \operatorname{Re} \beta)$ 
and $s$ and $r$ are real. Now

$$
\begin{aligned}
\operatorname{Re} \psi(i r, s) & =\operatorname{Re} \frac{s}{i r+\gamma}+\delta=\frac{s \operatorname{Re} \gamma}{|\gamma|^{2}+2 r \operatorname{Im} \gamma+r^{2}}+\delta \\
& \leqslant \delta-\frac{\operatorname{Re} \gamma\left[|\beta|^{2}-2 r \operatorname{Im} \beta+r^{2}\right]}{2 \operatorname{Re} \beta\left[|\gamma|^{2}+2 r \operatorname{Im} \gamma+r^{2}\right]} \\
& \equiv \frac{1}{D}\left[A+2 B r+C r^{2}\right],
\end{aligned}
$$

where $r$ and $s$ are real and

$$
\begin{aligned}
& A=2 \delta|\gamma|^{2} \operatorname{Re} \beta-|\beta|^{2} \operatorname{Re} \gamma, \\
& B=2 \delta \operatorname{Re} \beta \cdot \operatorname{Im} \gamma+\operatorname{Re} \gamma \cdot \operatorname{Im} \beta, \\
& C=2 \delta \operatorname{Re} \beta-\operatorname{Re} \gamma .
\end{aligned}
$$

If $\operatorname{Re} \gamma=0$ then $\delta=0$ and from (13) we obtain $\operatorname{Re} \psi(i r, s)=0$, for real $r$ and $s$.

Suppose $\operatorname{Re} \gamma>0$. We show that $\operatorname{Re} \psi(i r, s) \leqslant 0$ by using (13) and showing that $B^{2}-A C \leqslant 0$ and $C<0$. The condition $B^{2}-A C \leqslant 0$ is equivalent to

$$
W(\delta) \equiv 4 \delta^{2} \operatorname{Re} \beta \cdot \operatorname{Re} \gamma-2 \delta\left[|\gamma|^{2}+|\beta|^{2}+2 \operatorname{Im} \beta \cdot \operatorname{Im} \gamma\right]+\operatorname{Re} \beta \cdot \operatorname{Re} \gamma \geqslant 0 .
$$

We now show that $W(\delta) \geqslant 0$ for $0 \leqslant \delta \leqslant \delta_{0}$. From the identities

$$
|\beta+\gamma|^{2}-|\beta-\bar{\gamma}|^{2}=4 \operatorname{Re} \beta \cdot \operatorname{Re} \gamma
$$

and

$$
\begin{aligned}
|\beta|^{2}+|\gamma|^{2}+2 \operatorname{Im} \beta \cdot \operatorname{Im} \gamma & =|\beta+\gamma|^{2}-2 \operatorname{Re} \gamma \cdot \operatorname{Re} \gamma \\
& =\frac{1}{2}\left[|\beta+\gamma|^{2}+|\beta-\bar{\gamma}|^{2}\right]
\end{aligned}
$$

we find that the discriminant of (14) can be written in the form

$$
\Delta=|\beta+\gamma|^{2}|\beta-\bar{\gamma}|^{2} \geqslant 0 .
$$

A calculation shows that in (14) we have $W\left(\delta_{0}\right)=0$, where $\delta_{0}>0$ is given by (6). Combining this with $W(0)>0$ and $\Delta \geqslant 0$ we have $W(\delta) \geqslant 0$ for $0 \leqslant \delta \leqslant \delta_{0}$. Hence, by (14), $B^{2}-A C \leqslant 0$. From (15) we obtain

$$
0<|\beta+\gamma|-|\beta-\bar{\gamma}| \leqslant \operatorname{Re} \gamma
$$

and using this and $0 \leqslant \delta \leqslant \delta_{0}$ in the definition of $C$ we obtain

$$
C \leqslant 2 \delta_{0} \operatorname{Re} \beta-\operatorname{Re} \gamma=\frac{1}{4 \operatorname{Re} \gamma}\left[(|\beta+\gamma|-|\beta-\bar{\gamma}|)^{2}-4(\operatorname{Re} \gamma)^{2}\right]<0 .
$$

Hence $\operatorname{Re} \psi(i r, s) \leqslant 0$, and by applying Lemma 2 we obtain $\operatorname{Re} p(z)>0$. Since $p(z)=J(\beta, G(z))$, this implies that (11) is satisfied and hence that $G(z)$ is univalent.

We have shown that $G(z)$ is univalent in $U$. We will assume that $G(z)$ is univalent in $\bar{U}$. If not, we can continue the remainder of the proof with $G(r z), 0<r<1$, and obtain our final result by letting $r \rightarrow 1^{-}$, as was done in Theorem 1 . 
We now need to show that $F \prec G$. In order to do this we first introduce the function

$$
L(z, t) \equiv G(z)\left[(1+t) \beta z \frac{G^{\prime}(z)}{G(z)}+\gamma\right]^{1 / \beta},
$$

where $t \geqslant 0$. According to $(10), L(z, 0)=g(z)$. A simple calculation yields $L^{\prime}(0, t)$ $=[1+\beta t /(\beta+\gamma)]^{1 / \beta} \neq 0$ and

$$
z \frac{\partial L}{\partial z} / \frac{\partial L}{\partial t}=\gamma+(1+t) J(\beta, G)
$$

where $J(\beta, G)$ is defined by (3). Since $\operatorname{Re} \gamma \geqslant 0$, and since (11) has been shown, from (17) and Lemma 3 we conclude that $L(z, t)$ is a subordination chain. In particular, we have

$$
g(z)=L(z, 0) \prec L(z, t) \text { for all } t \geqslant 0 .
$$

Suppose $F \nprec 5 G$. Then there exists $z_{0} \in U$ such that $F\left(z_{0}\right)=G\left(\zeta_{0}\right),\left|\zeta_{0}\right|=1$ and $F\left(|z|<\left|z_{0}\right|\right) \subset G(U)$. Hence by Lemma 1 we have $z_{0} F^{\prime}\left(z_{0}\right)=(1+t) \zeta_{0} G^{\prime}\left(\zeta_{0}\right)$ with $t \geqslant 0$. From (9), (16) and (18) we obtain

$$
\begin{aligned}
f\left(z_{0}\right) & =F\left(z_{0}\right)\left[\beta \frac{z_{0} F^{\prime}\left(z_{0}\right)}{F\left(z_{0}\right)}+\gamma\right]^{1 / \beta}=G\left(\zeta_{0}\right)\left[(1+t) \beta \zeta_{0} \frac{G^{\prime}\left(\zeta_{0}\right)}{G\left(\zeta_{0}\right)}+\gamma\right]^{1 / \beta} \\
& =L\left(\zeta_{0}, t\right) \notin g(U),
\end{aligned}
$$

which contradicts the assumption $f \prec g$. Hence $F \prec G$ and the proof of Theorem 2 is complete.

Corollary 2.1. If $f, g \in H, g^{\prime}(0) \neq 0$ and

$$
\operatorname{Re}\left(1+\frac{z g^{\prime \prime}(z)}{g^{\prime}(z)}\right)>-\frac{1}{2}
$$

then

$$
f \prec g \Rightarrow \frac{1}{z} \int_{0}^{z} f(t) d t \prec \frac{1}{z} \int_{0}^{z} g(t) d t .
$$

Proof. If we let $\beta=\gamma=1$ then $K_{1,1}=H$, and from (6) and (7) we obtain $\delta=\delta_{0}=\frac{1}{2}$. Applying Theorem 2 with these parameters, we obtain the corollary.

Note that this result improves the result of D. Hallenbeck and S. Ruscheweyh [2, p. 192] who proved the conclusion with (19) replaced by $\operatorname{Re}\left(1+z g^{\prime \prime}(z) / g^{\prime}(z)\right)>0$.

REMARK. If $g$ is univalent in $U$ it is easy to show that (19) holds for $|z|<r_{0}=4$ $-\sqrt{13}$. Using $f\left(r_{0} z\right) \prec g\left(r_{0} z\right)$ from Corollary 2.1 we deduce that if $g$ is univalent in $U$ and $f \prec g$ then

$$
\frac{1}{z} \int_{0}^{z} f(t) d t \prec \frac{1}{z} \int_{0}^{z} g(t) d t
$$

at least for $|z|<4-\sqrt{13}=0.3944 \ldots$. This improves a result of R. Singh and S. Singh [11, Theorem 2] who proved this result for $|z|<2-\sqrt{3}=0.268 \ldots$.

We now apply Corollary 2.1 to obtain two mean-value results for regular functions. 
Result 1 . Taking $g(z)=1 /(1+z)^{2}$, we can easily deduce that $g^{\prime}(0) \neq 0$,

$$
\operatorname{Re}\left[1+z g^{\prime \prime}(z) / g^{\prime}(z)\right]=\operatorname{Re}[(1-2 z) /(1+z)]>-\frac{1}{2}
$$

and $G(z)=z^{-1} \int_{0}^{z} g(t) d t=1 /(1+z)$. Now using Corollary 2.1 we obtain

$$
f(z) \prec \frac{1}{(1+z)^{2}} \Rightarrow \frac{1}{z} \int_{0}^{z} f(t) d t \prec \frac{1}{1+z},
$$

when $f(z)$ is regular in $U$. Replacing $f$ by $f^{\prime}$ we obtain

$$
f^{\prime}(z) \prec \frac{1}{(1+z)^{2}} \Rightarrow \frac{f(z)}{z} \prec \frac{1}{1+z},
$$

or

$$
\operatorname{Re} \sqrt{f^{\prime}(z)}>\frac{1}{2} \Rightarrow \operatorname{Re} \frac{f(z)}{z}>\frac{1}{2} .
$$

This last result was obtained by Y. Komatu [3, Theorem 2] using a suitable Herglotz representation.

Result 2. If we take $g(z)=z / \sqrt{1+z^{2}}$, then $g^{\prime}(0) \neq 0, \operatorname{Re}\left[1+z g^{\prime \prime}(z) / g^{\prime}(z)\right]>$ $-\frac{1}{2}$ and from Corollary 2.1 we obtain

$$
f(z) \prec \frac{z}{\sqrt{1+z^{2}}} \Rightarrow \frac{1}{z} \int_{0}^{z} f(t) d t \prec \frac{z}{1+\sqrt{1+z^{2}}}=G(z),
$$

when $f(z)$ is regular in $U$. The set $g(U)$ is the domain containing the origin and bounded by the hyperbola $u^{2}-v^{2}=\frac{1}{2}$, while $G(U)=\{|w-1|<\sqrt{2},|w+1|<\sqrt{2}\}$. If we replace $f$ by $f^{\prime}$ we obtain

$$
f^{\prime}(z) \prec \frac{z}{\sqrt{1+z^{2}}} \Rightarrow \frac{f(z)}{z} \prec \frac{z}{1+\sqrt{1+z^{2}}}
$$

or

$$
\operatorname{Re}\left[f^{\prime}(z)\right]^{2}<\frac{1}{2} \Rightarrow\left(\left|\frac{f(z)}{z}-1\right|<\sqrt{2}\right) \text { and } \quad\left(\left|\frac{f(z)}{z}+1\right|<\sqrt{2}\right) .
$$

We close this paper with three additional distortion results that can be obtained from Theorem 2.

EXAMPLE. 1. If we choose $\beta=\gamma=\frac{1}{2}$ and $g(z)=z /(1+z)$, then the hypotheses of Theorem 2 are satisfied. Hence for $f \in K_{1 / 2,1 / 2}=\left\{f \in H, f(z)=z^{j} h(z), h(z) \neq 0\right.$, $j \geqslant 1$ \} we obtain

$$
\operatorname{Re} f(z)<\frac{1}{2} \Rightarrow \frac{1}{z}\left[\int_{0}^{z}\left[\frac{f(t)}{t}\right]^{1 / 2} d t\right]^{2} \prec \frac{4 z}{(1+\sqrt{1+z})^{2}}
$$

This last subordination implies the existence of a regular function $u(z)$ with $u(0)=0$ and $|u(z)|<1$ such that

$$
\frac{1}{z}\left[\int_{0}^{z}\left[\frac{f(t)}{t}\right]^{1 / 2} d t\right]^{2}=\frac{4 u(z)}{1+\sqrt{1+u(z)^{2}}} .
$$


If we let $v(z)=u(z) / z$ then $v(z)$ is regular in $U,|v(z)| \leqslant 1$ and we have

$$
\left[\int_{0}^{z}\left[\frac{f(t)}{t}\right]^{1 / 2} d t\right]^{2}=\frac{4 z^{2} v(z)}{(1+\sqrt{1+z v(z)})^{2}} .
$$

For a fixed $z_{0} \in U$ there exists a determination of $\sqrt{v\left(z_{0}\right)}=\zeta_{0} \in U$ such that

$$
\int_{0}^{z_{0}}\left[\frac{f(t)}{t}\right]^{1 / 2} d t=\frac{2 z_{0} \zeta_{0}}{1+\sqrt{1+z_{0} \zeta_{0}^{2}}}
$$

In particular, for $z_{0}=r, 0<r<1$, we have

$$
\int_{0}^{r}\left[\frac{f(t)}{t}\right]^{1 / 2} d t=\frac{2 r \zeta_{0}}{1+\sqrt{1+r \zeta_{0}^{2}}}
$$

The function $\psi(w)=2 r w /\left(1+\sqrt{1+r w^{2}}\right)$, with $w \in U$ and $0<r<1$, is convex and $\psi(\bar{U})$ is symmetric with respect to the real and imaginary axes (see Result 2 ). Hence from $(20)$ we deduce that $\operatorname{Re} f(z)<\frac{1}{2}$ implies

$$
\begin{aligned}
-2(\sqrt{2}-1) & \leqslant-2(\sqrt{1+r}-1) \leqslant \operatorname{Re} \int_{0}^{r}\left[\frac{f(t)}{t}\right]^{1 / 2} d t \\
& \leqslant 2(\sqrt{1+r}-1) \leqslant 2(\sqrt{2}-1)
\end{aligned}
$$

and

$$
-2 \leqslant-2(1-\sqrt{1-r}) \leqslant \operatorname{Im} \int_{0}^{r}\left[\frac{f(t)}{t}\right]^{1 / 2} d t \leqslant 2(1-\sqrt{1-r}) \leqslant 2,
$$

for $0<r \leqslant 1$. These results are sharp.

EXAMPLE 2. If we let $\beta=2, \gamma=1$ and $g(z)=z /\left(1+z^{2}\right)^{1 / 2}$ then the conditions of Theorem 2 will be satisfied. Hence if $f(z)$ is regular with $f(0)=0, f^{\prime}(0) \neq 0$, and $\operatorname{Re} z f^{\prime}(z) / f(z)>-\frac{1}{2}$ (i.e., $f \in K_{2.1}$ ) then

$$
f(z) \prec \frac{z}{\left(1+z^{2}\right)^{1 / 2}} \Rightarrow\left[\frac{1}{z} \int_{0}^{z} f^{2}(t) d t\right]^{1 / 2} \prec\left[1-\frac{\arctan z}{z}\right]^{1 / 2},
$$

which implies

$$
\operatorname{Re} f^{2}(z)<\frac{1}{2} \Rightarrow \frac{1}{z} \int_{0}^{z} f^{2}(t) d t \prec 1-\frac{\arctan z}{z}
$$

The function $p_{1}(z)=1-(\arctan z) / z=z^{2} / 3-z^{4} / 5+\cdots$ is bivalent, while the function

$$
p_{2}(z)=1-\frac{\arctan \sqrt{z}}{\sqrt{z}}=\frac{1}{2 \sqrt{z}} \int_{0}^{z} \frac{t}{1+t} t^{-1 / 2} d t
$$

is convex and univalent [8, Theorem 5]. Since $p_{2}\left(z^{2}\right)$ is also convex and since $p_{1}(z)=p_{2}\left(z^{2}\right)$, from $(21)$ we obtain the following sharp results: if $\operatorname{Re} f^{2}(z)<\frac{1}{2}$ and $F \in K_{2,1}$ then

$$
1-\frac{\operatorname{arctanh} \rho}{\rho} \leqslant \operatorname{Re} \frac{1}{z} \int_{0}^{z} f^{2}(t) d t \leqslant 1-\frac{\arctan \rho}{\rho}<1-\frac{\pi}{4} \quad \text { for }|z|=\rho<1 .
$$


For our final example we allow $\beta$ and $\gamma$ to assume complex values.

EXAMPLE 3. Let $\beta=e^{i \alpha}, \gamma=1-e^{i \alpha}$, with $\pi / 2<\alpha<\pi / 2$, and let $f, g \in K_{\beta, \gamma}$. From (6) and (7) we obtain

$$
\delta=\delta_{0}=(1-|2 \cos \alpha-1|) /(2-2|2 \cos \alpha-1|) .
$$

If $g$ satisfies (8) and $f \prec g$ then by Theorem 2 we have

$$
z\left[\frac{1}{z} \int_{0}^{z}\left(\frac{f(t)}{t}\right)^{e^{i \alpha}} d t\right]^{e^{-i \alpha}} \prec z\left[\frac{1}{z} \int_{0}^{z}\left(\frac{g(t)}{t}\right)^{e^{i \alpha}} d t\right]^{-i \alpha} .
$$

\section{REFERENCES}

1. G. M. Goluzin, On the majorization principle in function theory, Dokl. Akad. Nauk SSSR 42 (1953), 647-650. (Russian)

2. D. J. Hallenbeck and S. Ruscheweyh, Subordination by convex functions, Proc. Amer. Math. Soc. 52 (1975), 191-195.

3. Y. Komatu, On starlike and convex mappings of a unit circle, Kodai Math. Sem. Rep. 13 (1961), 123-126. MR 24A-253.

4. S. S. Miller and P. T. Mocanu, Second order differential inequalities in the complex plane, J. Math. Anal. Appl. 65 (1978) 289-305.

5. __ Differential subordinations and univalent functions, Michigan Math. J. 28 (1981), 157-171.

6 . Univalent solutions of Briot-Bouquet differential equations, J. Differential Equations (to appear).

7. S. S. Miller, P. T. Mocanu and M. O. Reade, Bazilevič functions and generalized convexity, Rev. Roumaine Math. Pures Appl. 23 (1974), 213-224.

8. __ Starlike integral operators, Pacific J. Math. 79 (1978), 157-168.

9. Ch. Pommerenke, Univalent functions, Vanderhoeck \& Ruprecht, Göttingen, 1975.

10. K. Sakaguchi, A note on p-valent functions, J. Math. Soc. Japan 14 (1962), 312-321. MR 26-6383.

11. S. Singh and R. Singh, Subordination by univalent functions, Proc. Amer. Math. Soc. 82 (1981), 39-47.

12. T. J. Suffridge, Some remarks on convex maps of the unit disk, Duke Math. J. 37 (1970), 775-777.

Department of Mathematics, State University of New York, BrockPort, New York 14420

Faculty of Mathematics, Babes - Bolyai University, 3400 Cluj - Napoca, Romania

Department of Mathematics, University of Michigan, AnN Arbor, Michigan 48109 